\title{
Guerre et journalisme culturel : les variantes du "feuilleton" durant la Première Guerre
}

Krieg und Kulturjournalismus: das Feuilleton im Ersten Weltkrieg

War and cultural journalism: the variations of the 'column' during the First

World War

\section{Françoise Knopper}

\section{(2) OpenEdition}

\section{Journals}

Édition électronique

URL : http://journals.openedition.org/ceg/1945

DOI : $10.4000 /$ ceg. 1945

ISSN : 2605-8359

Éditeur

Presses Universitaires de Provence

Édition imprimée

Date de publication : 15 juin 2014

Pagination : 53-76

ISSN : 0751-4239

Référence électronique

Françoise Knopper, «Guerre et journalisme culturel : les variantes du "feuilleton" durant la

Première Guerre », Cahiers d'Études Germaniques [En ligne], 66 | 2014, mis en ligne le 17 décembre

2017, consulté le 19 novembre 2020. URL : http://journals.openedition.org/ceg/1945 ; DOI : https:// doi.org/10.4000/ceg.1945 


\title{
Guerre et journalisme culturel : les variantes du "feuilleton" durant la Première Guerre
}

\author{
Françoise KNOPPER
}

CREG, université de Toulouse-Le Mirail

En consultant un journal, quotidien ou hebdomadaire, paru en Allemagne entre 1914 et 1918, on trouvera, à la une, des informations sur les opérations militaires puis, trois ou quatre pages plus loin, le « feuilleton », lequel se repèrera aisément car il figure sous un double trait épais et est généralement situé en bas de page. Ces feuilletons sont ainsi présentés comme des articles marginaux, au sens propre comme au figuré, destinés à détendre les lecteurs. Autrement dit, les rédactions et les lecteurs des journaux ont tenu à préserver, même durant la Première Guerre, cette rubrique qui était entrée dans les usages culturels depuis quelque 150 ans ${ }^{1}$. Le genre du «feuilleton » avait en effet connu plusieurs heures de gloire par le passé : les feuilletonistes avaient par exemple répondu aux attentes des lecteurs à l'époque de l'Aufklärung en sachant à la fois distraire et éduquer; puis les auteurs de la Jeune Allemagne avaient utilisé cette rubrique dans les années $1830-1840$ pour s'exprimer tout en prenant une position de repli culturel, par exemple Heine, Börne, Laube, Gutzkow $^{2}$; ils étaient largement diffusés à la fin du XIX ${ }^{\mathrm{e}}$ siècle et les Wanderungen durch die Mark Brandenburg ont permis à Fontane d'aiguiser son talent d'écrivain. Le mode de la narration, toutes périodes confondues, se devait d'être plaisant, léger et élégant ${ }^{3}$. Comme en France, la rubrique du feuilleton pouvait parfois consister en une suite d'épisodes, mais elle

\footnotetext{
${ }^{1}$ Après avoir été quelque temps délaissé, le genre du feuilleton culturel a connu un regain d'intérêt grâce à Almut TODOROW (Das Feuilleton der Frankfurter Zeitung in der Weimarer Republik, Tübingen, Niemeyer, 1996) et aux travaux menés sous son impulsion. Cf. aussi l'état de la recherche chez Christina PRÜWER, Willy Haas und das Feuilleton der Tageszeitung Die Welt, Würzburg, Königshausen \& Neumann, 2007, p. 17-20, et Hildegard KERNMEYER, «Perspektiven der Feuilletonforschung: Vorwort », Zeitschrift für Germanistik, vol. XXII, n 3, 2012, p. 494-508.

2 Ernst ECKSTEIN, Beiträge zur Geschichte des Feuilletons, Leipzig, Hartknoch, 1876, p. $70-107$.

3 TODOROW, Das Feuilleton der Frankfurter Zeitung, p. 260.
} 
proposait le plus souvent un article autonome, relevant de la catégorie des formes narratives brèves et pouvant consister en un reportage, un compte rendu, un essai, une nouvelle ${ }^{4}$. De tels articles, tout en empruntant leur cadre formel au discours journalistique, avaient des ambitions littéraires ${ }^{5}$ : tout en s'insérant dans le quotidien, ils le transposaient et l'interprétaient; ils pouvaient se référer à des faits mais les esthétisaient.

La majorité des exemples qui vont être évoqués dans la présente étude sont des reportages rédigés par des journalistes qui ont été dépêchés sur le front par leurs rédactions, ou ont demandé à l'être ; ils ne font partie ni des militaires - dont le déplacement relevait évidemment du devoir - ni de touristes dont les déplacements n'auraient pas répondu à une mission. Tandis que l'on peut encore parler de tourisme chez les militaires visitant les Balkans en 1912 et 1913, cette notion paraît plus contestable durant les années 14$18^{6}:$ les reportages se présentent dorénavant comme des écrits semi-littéraires et semi-privés, ils associent réalisme et recherche stylistique, idéologie et observations de terrain. C'est pourquoi une des questions qui se posent est de savoir comment les feuilletonistes ont géré la touche de raffinement inhérente au feuilleton culturel dans un environnent où planaient la tension, l'angoisse, le danger. Que pouvaient apporter de tels intermèdes narratifs et culturels dans le contexte des conflits militaires? Comblaient-ils un déficit informatif? Confrontés aux difficultés morales et matérielles de la guerre, avaient-ils des particularités thématiques ? Ce double trait qui, sur le papier, isolait le feuilleton des autres articles, était-il un moyen commode que la rédaction du journal pouvait utiliser pour élargir sa marge de manœuvre, s'accorder un peu de liberté malgré la censure ? La porosité des deux registres, celui du discours journalistique et celui de l'évasion esthétique, plaçait-elle les auteurs dans un entre- deux, leur servait-elle de garde-fou, ou bien toute neutralité était-elle au contraire incompatible avec ces temps de guerre?

Des exemples d'articles parus respectivement dans de grands organes de presse (Frankfurter Zeitung, Berliner Tageblatt, Neue Freie Presse, Die Weissen Blätter) vont nous permettre d'examiner des stratégies de communication et d'écriture utilisées dans des feuilletons. Ces exemples ne sont pas tirés de la presse officielle («Kriegspresse ») ni de gazettes de partis politiques, mais - dans les limites de cet article - de quelques représentations

4 Sur la diversité des feuilletons, cf. Wilmont HAACKE, dont on utilisera plutôt le Handbuch des Feuilletons, Emsdetten, Lechte, 1952, ou la contribution au Handbuch der Publizistik d'Emil Dovifat, t. II, Berlin, de Gruyter, 1969, plutôt que Feuilletonkunde, Leipzig, Hiersemann, 1943, son travail d'habilitation, qui avait été très marqué par l'arrière-plan de l'époque. Cf. aussi Heinz KNOBLOCH, Vom Wesen des Feuilleton, Halle (Saale), VEB Sprache und Literatur, 1962.

5 Norbert BACHLEITNER, « Littérature industrielle : Bericht über Untersuchungen zum deutschen und französischen Feuilletonroman im 19. Jahrhundert ", Internationales Archiv für Sozialgeschichte der deutschen Literatur, vol. XIX, 1994, $\mathrm{n}^{\circ}$ spécial 6, p. 159-223.

${ }^{6}$ Mechthild GOLCZEWSKI, dans Der Balkan in deutschen und österreichischen Reise- und Erlebnisberichte 1912- 1918, Wiesbaden, Steiner, 1981, établit une distinction entre ces deux catégories en analysant plusieurs témoignages d'officiers. 
de scènes de guerre transposées dans cette rubrique. Leurs paradigmes n'étant pas ceux de tacticiens ou d'historiens, ils viennent illustrer une « autre face de 1'histoire » de la Grande Guerre?.

Les auteurs n'en demeuraient pas moins tributaires de la censure et de l'attitude des autorités en matière de liberté d'expression, et les années de parution des articles seront à relier aux conditions dans lesquelles la presse pouvait travailler en temps de guerre ${ }^{8}$. Il faut à cet égard différencier la situation entre l'Allemagne et l'Autriche-Hongrie. En Autriche', la censure fut confiée d'emblée à des autorités militaires, comme en France, et la censure ne se relâcha qu'au début de l'année 1917. En Allemagne en revanche, la situation évolua au fil des ans ${ }^{10}$ et les hésitations des autorités semblent avoir quelque peu fragilisé l'efficacité des contrôles sur ce plan, de sorte que, quand les feuilletonistes apporteront un appui explicite à l'effort de guerre, ce ne sera pas à la seule censure qu'il faudra l'imputer et d'autres facteurs seront à rechercher. Comme la censure dépendit tout d'abord de civils du service des affaires étrangères (« Presseamt der Politischen Abteilung des Auswärtigen Amts ») car, à cette époque, le chancelier Bethmann-Hollweg privilégiait le consensus, l'union sacrée, ce n'est sans doute pas par hasard que fut publié à ce moment-là un article ironique dans les Weisse Blätter, mensuel qui paraissait encore à Leipzig : dans cet article intitulé « Der erlöste Feuilletonist », Christian Heinold feignait, sur le ton de la dérision, de se féliciter du fait que les feuilletonistes aient dorénavant la tâche facile puisqu'il leur était demandé de préserver l'union sacrée et que leurs commentaires pourraient faire l'éloge de poètes nationalistes sans plus subtiliser, sans réserver leur jugement, au nom du prétendu « véritable art allemand $»^{11}$. Puis, en un deuxième temps, à partir d'octobre 1915, la censure fut réorganisée et confiée à des militaires (« Kriegs-Presse-Amt im Stellvertretenden Generalstab ») et la situation se durcit davantage lors de l'instauration de la dictature militaire de Ludendorff en 1916 ; la tâche des censeurs, durant cette deuxième phase, consistait à atténuer ou lisser les différences entre l'armée, les partis politiques et la population civile ; c'est

\footnotetext{
${ }^{7}$ Pour reprendre le titre de Klaus AMANN et Hubert LENGAUER (dir.), Österreich und der Große Krieg 1914-1918. Die andere Seite der Geschichte, Wien, Brandstätter, 1989.

8 Cf. Martin CREUTZ, Die Pressepolitik der kaiserlichen Regierung während des Ersten Weltkriegs, Bern, Peter Lang, 1996 ; dans le cadre restreint de notre article, il n'est pas possible d'établir des comparaisons avec des pratiques à l'étranger mais nous signalons une mise en perspective de la censure en France et en Allemagne chez Thilo EISERMANN, Pressephotographie und Informationskontrolle im Ersten Weltkrieg. Deutschland und Frankreich im Vergleich, Hamburg, Kämpfer, 2000, p. 46 et suiv. Et, pour la littérature, AnneMarie SAINT-GiLlE et Jean-Jacques POLLET (dir.), Ecritures franco-allemandes de la Grande Guerre, Arras, Artois Presses Université, 1996.

9 Petronilla Ehrenreis, Kriegs- und Friedensziele im Diskurs. Regierung und deutschsprachige Öffentlichkeit Österreich-Ungarns während des Ersten Weltkriegs, Wien, Institut für Geschichte, 2005.

${ }^{10}$ Kurt KosZYK, Die Pressepolitik im Ersten Weltkrieg, Düsseldorf, Droste, 1968.

${ }^{11}$ La raillerie de C. HeINOLD concerne Bloem et Herzog (cf. Die Weissen Blätter, $2^{\mathrm{e}}$ année, $\mathrm{n}^{\circ} 2$, janvier 1915, p. 128-129).
} 
durant cette période que par exemple les reportages de Norbert Jacques, dont nous parlerons plus loin, sont publiés dans la presse. Enfin un relatif assouplissement de la censure est concédé à l'hiver 1917 dans la mesure où ce fut à la chancellerie qu'on créa un service de presse, et ce changement est souhaité par un périodique tel que le Berliner Tageblatt.

En somme, comme le souligne Gerd Krumeich ${ }^{12}$, la situation de la presse n'était pas celle qu'elle connaîtra sous Hitler lors de la « guerre totale ». Les feuilletonistes pouvaient gérer en connaissance de cause les relations entre le lieu des combats, quand ils les observaient, et « le front de l'arrière » (« Heimatfront»), pour qui ils écrivaient. Nul doute que les soldats avaient besoin du soutien de l'arrière et que les civils étaient incités à manifester leur solidarité. Nous le verrons d'abord en montrant que les auteurs de ces feuilletons veillent à être "des agents de liaison ${ }^{13}$ ", à transmettre les informations qu'ils ont pu se procurer en enquêtant eux-mêmes sur place, et qu'ils s'efforcent de rendre visible une factualité qui est étrangère aux lecteurs, à ceux de l'arrière. Puis nous examinerons en quoi certains de ces feuilletons vont plus loin et se placent délibérément au service d'une propagande idéologique, le journaliste prolongeant le conflit des armes par la guerre des mots, guerre dont il est un acteur essentiel; les exemples de propagande tendancieuse que nous avons retenus ici ont été écrits en faveur de l'Allemagne. Ces exemples seront ensuite complétés par des feuilletons attestant des positions intermédiaires, patriotiques mais non bellicistes, celles de journalistes qui osèrent cependant parler de paix mais sans basculer dans le militantisme d'auteurs qui, pacifistes patentés, faisaient quant à eux usage de techniques de représentations expressionnistes et étaient publiés dans des revues littéraires.

\section{Focalisation sur l'enquêteur}

Les différences entre une chronique officielle, dont la vocation se voulait historique et militaire, et des reportages de guerre publiés en tant que feuilletons étaient notables. C'est dû en particulier à la focalisation sur le témoignage individuel de feuilletoniste, tandis que les autres catégories de chroniqueurs, ceux qui ont été commandités par l'armée, relatent les opérations sans en référer à leur propre personne et ils semblent s'effacer derrière l'ampleur des événements qu'ils ont été chargés de consigner.

\footnotetext{
12 Dans son introduction au volume : Gerhard HIRSCHFELD (dir.), Kriegserfahrungen. Studien zur Sozial- und Mentalitätsgeschichte des Ersten Weltkriegs, Essen, Klartext, 1997, p. 12 et suiv.

${ }^{13}$ Cf. Almut Lindener-Wirsching, « Patrioten im Pool ? Deutsche und französische Kriegsberichterstatter im Ersten Weltkrieg », in Ute DANIEL (dir.), Augenzeugen. Kriegsberichterstattung vom 18. zum 21. Jahrhundert, Göttingen, Vandenhoeck \& Ruprecht, p. 113-140.
} 
Le supplément hebdomadaire au Berliner Lokalanzeiger, Deutsche Kriegszeitung, publiait par exemple les articles du contre-amiral von Schliepe et les accompagnait des clichés de photographes officiels ${ }^{14}$. La une comporte ainsi systématiquement le portrait d'un grand général allemand, l'empereur Guillaume II figure sur le numéro 1 daté du dimanche 16 août 1914. Le titre de cette chronique est programmatique «Aus großer Zeit. Von einem alten preußischen Offizier » et la rédaction recommande aux lecteurs de conserver tous les numéros afin de se construire, en quelque sorte en direct, la mémoire de «l'hérö̈que combat mené par la nation » («Denkmal des Heldenkampfes unseres Volkes »). Le périodique n'entend donc nullement publier un feuilleton mais éditer des annales rédigées par un technicien de la guerre et pour servir à la postérité. Comme c'est seulement la raison d'État qui compte ici, la propagande s'opère par l'image - les progrès techniques étant conséquents à cet égard - tout comme par le texte : soulignant que le moment n'est pas venu de songer à la paix, l'auteur relate les victoires, en particulier celles de Hindenburg, conteste ce qu'il estime relever de la désinformation de la part des ennemis, dénonce des atrocités commises dans le camp adverse. C'est dans cet esprit qu'il est aussi périodiquement fait allusion au patrimoine culturel allemand, par exemple Goethe est mis à contribution dans le numéro du 12 décembre 1915 :

Wir werden uns mit der Zeit an den Gedanken gewöhnen müssen, daß unseren Feldgrauen in diesem Weltkriege keine geographische Grenze mehr gezogen ist. Was würde der Dichter des Faust dazu gesagt haben, wenn man ihm erzählt hätte, daß nicht nur hinten, fern in der Türkei, nein selbst in Arabien deutsche Fäuste tätig seien! 15

D'ailleurs la signature "Par un vieil officier prussien » est peut-être un hommage aux usages internes des militaires prussiens car elle rappelle les entrefilets que le roi Frédéric II inséra jadis lui-même dans la presse et qu'il signa de façon analogue en tant qu'officier anonyme.

Les feuilletons se présentent différemment d'une telle chronique. Cela commence par le montage des articles : à la différence de la Kriegszeitung dont la typographie était pensée à partir des articles du «vieil officier prussien », les feuilletons ne sont pas placés plastiquement au pinacle, même s'ils sont eux aussi thématiquement liés à l'esprit des périodiques. En outre, au lieu de l'ambition prioritaire que le chroniqueur avait de servir à la postérité, le feuilletoniste est un journaliste qui livre ses impressions dans le cadre d'un instant rapproché. À cela s'ajoute le fait que ses informations se

\footnotetext{
${ }^{14}$ Le texte a été numérisé et est consultable sur le site de l'université de Heidelberg. Pour l'année 1914 : http://digi.ub.uni-heidelberg.de/diglit/feldztgdkz1914.

${ }^{15}$ Les journalistes français le lisaient et y répliquaient puisque Le Temps le cite et le conteste, le 2 février par exemple : «Le contre-amiral Schliepe, dans le Lokal-Anzeiger, soutient la même thèse "Nos sous-marins puissants, écrit-il, répandent la terreur ; n'hésitons-pas à les employer contre tout navire battant pavillon britannique. Peu importe ce que pensent les personnes voyageant sous ce pavillon" ".
} 
veulent certes authentiques mais que le reporter ne s'arroge pas la stature d'un expert militaire et que, le cas échéant, il s'abrite derrière les avis qu'il dit récolter sur place. Enfin, ce sont précisément son approche individuelle, son expérience, ses sentiments, son identité, qui sont mis en exergue.

En dépit de ces différences dans les intentions et les orientations que l'on constate entre les annales d'un tacticien et les feuilletons des journalistes, l'objet décrit est le même, la guerre, si bien que des points communs sont à relever, d'autant que la gravité des enjeux n'est pas perdue de vue même quand les recherches esthétiques sont très travaillées par les auteurs des articles. Les feuilletons qui se rapprochent le plus des chroniques sont les reportages qui précisent la localisation des régiments observés de façon hyperdétaillée. Prenons les exemples d'Alice Schalek et de Norbert Jacques : leurs enquêtes livraient en quelque sorte une photographie de l'environnement, et étaient susceptibles de contribuer à étancher la soif d'information du public.

On ne saurait être surpris si les noms des officiers ne sont que rarement indiqués dans ces reportages, étant donné que c'était là une exigence de la part des autorités : il était interdit de désigner nommément les chefs militaires rencontrés. En revanche, les données géographiques sont précisées, il n'est pas exceptionnel que des cotes cartographiques soient même indiquées. À première vue, l'objectif en est d'aider le lecteur de l'époque à repérer le trajet du reporter et à suivre son évolution sur des cartes, sachant que des articles précédents ont justement traité de ces aspects militaires. La localisation confirme en outre l'authenticité du témoignage, laquelle peut être certifiée par l'emplacement des régiments observés. Mais, dans un feuilleton, cette reconstruction de la topographie débouche en définitive sur la construction d'une autre catégorie de paysage, d'un horizon virtuel ${ }^{16}$, qui pouvait servir à stabiliser la représentation et circonscrivait au moins dans l'espace les limites d'un conflit qui, lui, s'enlisait dans l'espace et la durée. Tout comme d'autres indications concrètes récurrentes dans tous ces textes - départ des trains, uniformes des infirmières -, de tels repères semblaient garantir une cohérence et assurer une continuité par rapport à des choses connues.

Du 4 avril au 15 septembre 1916, la journaliste viennoise Alice Schalek (1874-1956), une des rares femmes reporters de guerre, publie dans la Neue Freie Presse ${ }^{17}$ une série d'articles sur le front qu'elle avait visité durant plusieurs semaines dans les Dolomites et le long de l'Isonzo, dans la Slovénie actuelle. D'un bout à l'autre, elle fait montre d'un patriotisme indéfectible. À

\footnotetext{
${ }^{16}$ Bernd HÜPPAUF, « Räume des Destruktion und Konstruktion vom Raum. Landschaft, Sehen und Raum im Ersten Weltkrieg ", Krieg und Literatur/ War and Literature. Internationale Beträge zur Erforschung der Kriegs- und Antikriegsliteratur, vol. III, n 5/6, 1991, p. 105-123, ici p. 109.

Cf. Adam WANDRUSZKA, Geschichte einer Zeitung : Das Schicksal der Presse und der Neuen Freien Presse von 1948 bis zur Zweiten Republik, Wien, Neue Wiener Presse, 1958; Sigurd SCHEICHL, « Journalisten leisten Kriegsdienst, Die Neue Freie Presse im September 1915 », in AMANN et LeNGAUER (dir.), Österreich und der Große Krieg 1914-1918, p. 104-108.
} 
cela s'ajoute dans son propre cas un civisme féministe que la guerre lui permet d'afficher puisqu'elle souligne qu'elle ne s'est pas contentée d'observer l'endurance - jusqu'à l'épuisement extrême - des soldats mais qu'elle a partagé leurs efforts physiques :

Die Höhe treibt uns den Schweiß aus den Poren. Die Sonne macht aus dem
"Laufgraben" einen Hexenkessel. Eine Erlösung scheint es, daß Tolmein immer
näher rückt. Die eigene Ermüdung drängt mir auf die Zunge: "Tragen die Leute
auch über diesen Weg die Kochkisten, die Munition, das Bauholz hinauf?" Und
dabei zuckt es wie eine heimliche Erleichterung durch meine Gedanken, daß ich
selbst da nur hinunter-, nicht hinaufsteigen muß. "Aber früher galt es, den Mrzli
und den Vodil ganz ohne Weg und ohne Deckung zu erklettern! Dieser Steig ist
doch ganz im Feuer, im Finstern gehauen worden. Und im Tragen, im Bauen
kämpften die Männer noch!"
Dieses schauerliche Wort "früher" ist im Isonzowortschatz das ärgste. Wie muß
es "früher" gewesen sein. Müde sind wir und es ist jämmerlich heiß. ${ }^{18}$

Ce monologue intérieur a ceci de caractéristique qu'il montre comment il s'opérait, ici et dans le reste du reportage, un transfert du courage des combattants sur le reporter de guerre, A. Schalek fut d'ailleurs décorée en 1917 du « Goldenes Verdienstkreuz » de la monarchie austro-hongroise pour sa bravoure. Comme beaucoup d'autres reporters, elle aspire à partager l'effort, à s'assimiler aux soldats. Chaque journaliste en trouvait son identité individuelle enrichie, ce qui dans le cas d'une femme se dégageait plus fortement encore que chez d'autres. Mais ce n'est pas cet aspect identitaire que Karl Kraus retiendra dans Die letzten Tage der Menschheit où, avec force sarcasmes, il reprochera à A. Schalek de s'être inféodée à l'esprit du temps, d'avoir soutenu le militarisme ambiant, exploité la guerre et dévoyé le discours journalistique à des fins commerciales ${ }^{19}$. Outre le soin mis à localiser les événements, les journalistes transposent presque toujours leurs impressions sous forme d'instantanés, tant et si bien qu'on ignore la plupart du temps le décalage entre le moment de la publication et le déroulement des opérations. Or - rétrospectivement, pour le lecteur d'aujourd'hui - ce décalage était un révélateur de la durée de la guerre. Ainsi, comme les affrontements sur le front célèbre de l'Isonzo firent des milliers de morts et se déroulèrent durant plus de deux ans, du 23 juin 1915 au 10 novembre 1917, la description d'A. Schalek peut alimenter notre analyse de ce qu'était une guerre de montagnes et montrer les difficultés qu'il y eut à mettre un terme à ces opérations dans les Dolomites. Mais, prisonnière qu'elle était des contingences du moment, la journaliste a quant à elle signalé la moindre avancée des soldats autrichiens; sans véhiculer des convictions politiques, le seul fait de sélectionner dans ce qu'elle voyait et entendait ce qui était

18 Alice SCHALEK, Neue Freie Presse, 15 septembre 1916.

19 Cf. Irina DJASSEMY, Die verfolgende Unschuld. Zur Geschichte des autoritären Charakters in der Darstellung von Karl Kraus, Wien, Böhlau, 2011, p. 12 et suiv. 
susceptible d'inciter à l'optimisme, d'affirmer que la situation était «meilleure qu'avant», l'impliquait dans le spectre de la guerre.

Même si le ton peut sembler anecdotique, la curiosité de ce genre de reporters n'est pas celle d'un touriste. La majorité d'entre eux justifient la rédaction de tels articles par le devoir journalistique d'informer. Un tel devoir ne résidait pourtant pas dans la vérification des données officielles ni dans la transmission de détails tactiques : il s'agissait pour ces auteurs de se rendre au cœur de l'actualité et de décrire le quotidien, surtout dans les tranchées, d'avoir un contact immédiat avec la guerre, de la découvrir.

Un revers de la médaille est que ces auteurs-là ne cherchaient alors pas à expliquer la guerre, ils la recevaient comme une donnée. A. Schalek en livre un témoignage au tout début de sa série d'articles. Entendant une cliente dans une librairie viennoise qui se refusait à acheter un livre parlant de la guerre et s'exclamait «S'il vous plait, rien sur la guerre!» (« Bitte, nichts vom Krieg! »), la journaliste s'indigne :

Nein, ich meine, man hört nicht genug, weiß viel zu wenig von ihm. Die
furchtbar knappen Worte, in die man ihn für uns zusammengepresst hat, stehen
wie eine Mauer vor uns. Schlacht! -Welch ein Ausdruck. Sieg! - Welch eine
Zusammenfassung. Daß dahinter Menschen sterben - wissen wir's? Daß jedes
dieser Worte Tausende von Schicksalen umfasst - würdigen wir's? Während der
Krieg Hunderttausende von Leben umkrallt und mitten aus ihnen heute den,
morgen jenen verbluten lässt - sollen wir uns eher die Ohren zuhalten, damit wir
ungestörter lachen oder träumen können, damit wir sagen können "Bitte, nichts
vom Krieg?".

En rejetant l'abstraction, en rendant visible et tangible ce qu'est un conflit, sans se borner à utiliser des mots, le reportage de guerre entendait être la tâche la plus noble qui pouvait incomber à ces journalistes enquêteurs s'ils se chargeaient de relier le front aux civils de l'arrière. Parce que « la guerre est là », il y aurait selon cette journaliste un impératif de vérité, de camaraderie et de civisme. Malgré les termes connotés positivement de "Schlacht» et «Sieg », sa formule « Der Krieg ist da » ne marque pas d'euphorie ${ }^{21}$, elle est plutôt le constat d'un cas de force majeure, l'écho d'une réalité qui serait incontournable. Néanmoins, comme cette journaliste affirme avec fougue que l'élite cultivée ne devrait pas l'éluder, elle rend ténue - volens nolens - la frontière entre résignation et enthousiasme.

La narration à la première personne confère aux reportages une part de spontanéité et de suspense. Cela ne conduit pas forcément à exalter la guerre, comme on le voit chez A. Schalek, mais cela peut le faciliter et servir de tremplin, comme nous allons le voir dans les articles de Norbert Jacques. Tout un chacun a certainement déjà croisé une fois le nom de Norbert Jacques

\footnotetext{
${ }^{20}$ Alice SCHALEK, Neue Freie Presse, 6 avril1916.

${ }^{21}$ Sur les signes de l'acceptation de la guerre par la presse autrichienne, sans qu'elle l'ait majoritairement accueillie avec enthousiasme, cf. EHRENREIS, Kriegs- und Friedensziele im Diskurs, p. 381.
} 
car c'est lui qui avait écrit en 1921 Dr. Mabuse, der Spieler, texte dont Fritz Lang s'inspira en 1922. Les germanistes connaissent souvent aussi le scénario qu'il a écrit en 1940 pour un film nationaliste sur Schiller. Né à Luxembourg en 1880, N. Jacques mourut en 1954 à Coblence. Journaliste et romancier prolifique, il opta très tôt pour la langue et la culture allemandes. Il a entrepris de nombreux voyages et séjourné plus d'un an à Paris, en 1910 et 1911, y fréquentant journalistes et artistes, nouant des liens amicaux avec François de Curel, René Schickele et Marcel Ray ${ }^{22}$. Ses reportages écrits entre 1914 et 1916 ont été publiés dans les pages culturelles de périodiques tels que Frankfurter Zeitung, Neuer Merkur, Gartenlaube, ils font œuvre de propagande en faveur de la germanité. Simultanément, ces reportages ont été regroupés par $\mathrm{N}$. Jacques dans trois livres - tirés à 17000 exemplaires, édités et réédités à Berlin chez $\mathrm{S}$. Fischer ${ }^{23}-$, publication qui lui permit de lier un discours journalistique portant sur l'actualité à son ambition explicite de s'engager et de transmettre des convictions durables ${ }^{24}$. Sous sa plume aussi, le genre du feuilleton se prête à diffuser des observations notées sur le vif ainsi que des anecdotes. Sa manière de les relater met particulièrement en relief la composante du déplacement individuel car tout se passe comme si le reporter bougeait sans arrêt : les moyens de transport ont des retards, il y a moult imprévus. L'aspect fragmentaire et discontinu de la parution dans des périodiques accompagnait la mobilité du reporter et facilitait la lecture, d'autant plus que l'emplacement des lieux est repérable ici encore, N. Jacques étant allé sur trois fronts. Dans les articles rassemblés dans Die Flüchtlinge. Von einer Reise durch Holland hinter die belgische Front (1915), N. Jacques rapporte comment, partant de la Suisse où il habite, il se rend en Belgique au moment où commence la bataille d'Anvers (le lecteur pourra donc situer ce déplacement entre le 28 septembre et le 10 octobre 1914); il propose ses services en tant qu'espion, puis, comme son offre est caduque, il reste pour rassembler de la documentation sur le déroulement de la guerre. Dans London und Paris im Krieg. Erlebnisse auf Reisen durch England und Frankreich in Kriegszeit (1915), il relate la suite : de Belgique, il s'embarque début novembre pour Londres, puis voyage à travers la France; il se présente tantôt comme journaliste de la Frankfurter Zeitung tantôt comme simple touriste suisse. Il y reste environ trois mois, durant l'automne et l'hiver 1914, et mène dans les deux pays une enquête sur les mentalités ainsi que sur le nombre des

\footnotetext{
${ }^{22}$ Norbert JACQUES avait donné en 1950 des précisions factuelles dans l'autobiographie Mit Lust gelebt, rééditée depuis, St. Ingbert, Röhrig, 2004. Une présentation de son œuvre figure dans la monographie de Günter SCHOLDT, Der Fall Norbert Jacques. Über Rang und Niedergang eines Erzählers (1880-1954), Stuttgart, Heinz, 1976.

${ }^{23}$ Norbert JACQUES, Die Flüchtlinge. Von einer Reise durch Holland hinter die belgische Front, Berlin, S. Fischer, 1915, 108 p. ; Norbert JACQUES, London und Paris im Krieg Erlebnisse auf Reisen durch England und Frankreich in Kriegszeit, Berlin, S. Fischer, 1915, 211 p. ; Norbert JACQUES, In der Schwarmlinie des österreichisch-ungarischen Bundesgenossen, Berlin, S. Fischer, 1916, 131 p.

24 "Ich widme sie [die Reiseberichte nach London und Paris] in Liebe und Ehrfurcht meiner Wahlheimat Deutschland» (London und Paris).
} 
soldats chez l'ennemi (estimant que le nombre est bien moins important qu'on ne le croyait). Pour finir, il se rend à Vienne et obtient l'autorisation d'aller sur le front de l'Est, en Podolie (Ukraine), entre Bug et Dniepr ; il y est envoyé en tant que reporter de guerre par la Frankfurter Zeitung. Il en résulte la relation In der Schwarmlinie des österreichisch-ungarischen Bundesgenossen (1916).

Ce qui frappe en outre, quel que soit le reportage de guerre de N. Jacques, est la pérennisation de ses pratiques en matière de civilité. Il reste un bourgeois riche, voyage en première classe, fréquente les meilleures tables ou les hôtels luxueux, tel un aventurier doublé d'un dandy. Il privilégie des lieux où les cultures sont en contact : bateau, train, cafés. La capacité de s'entretenir avec des personnes d'origines géographiques diverses implique selon lui la «bienséance $e^{25}$ » des propos échangés. Cette prédilection atteint un point d'orgue à la fin de son troisième livre : la scène se déroule à Czernowtiz dans le "café Europe », nom programmatique : un pope y joue avec un libraire juif et le député roumain, N. Jacques lui-même déjeune avec un Kommerzialrat de Bucovine auquel il prend le temps de démontrer en quoi les liens de la monarchie austro-hongroise avec l'Allemagne seraient bénéfiques aux économies.

Par ailleurs, N. Jacques aime décrire les mouvements de masse et recourir alors à un style épique. Les transports des blessés et les ambulances de la Croix Rouge composent une sorte de ballet monotone. Les flots de réfugiés belges qui sont évacués d'Anvers sont les premiers à stimuler son inspiration car il a l'impression d'assister à un gigantesque exode («Völkerwanderung ») : on a l'impression de lire un scénario de film. Un moment particulièrement saisissant est celui où, avançant à contre-courant, $\mathrm{N}$. Jacques tente de gagner le lieu où la bataille se livre et de remonter le défilé des Belges en fuite. Mais il est finalement balayé, emporté malgré lui par le flot des réfugiés et, au lieu de rejoindre les soldats allemands, il se retrouve dans un train pour la Hollande.

$\mathrm{Au}$ total, la mobilité est l'élément structurant de chaque reportage, le narrateur restant le seul repère et forçant ainsi le lecteur à suivre son regard. Cette focalisation est d'autant plus lourde de sens si le journaliste tente d'imposer ses vues nationalistes.

\section{L'affrontement par les mots ou quand le patriotisme se mue en propagande}

L'affirmation du patriotisme étant omniprésente, la part d'autocensure exercée par les rédactions est à prendre en compte ; selon Wolfgang

25 « zwischen gesitteteten Menschen » (JACQUES, London und Paris, p. 36). 
H. Mommsen ${ }^{26}$ elle était même plus efficace que la censure exercée par les autorités officielles. De surcroît, si, faute d'optimisme, le journaliste avait insisté sur le nombre des morts et les destructions, il aurait risqué de passer pour un traître ou un défaitiste, car, en temps de guerre, de telles informations sur l'état des mentalités auraient été exploitables par le camp adverse ${ }^{27}$. Mais le discours ne se mue en propagande à proprement parler que si le feuilletoniste pratique un véritable conflit verbal et entretient la " guerre des esprits $^{28} »$. C'est le cas de N. Jacques dont le nationalisme germanique exacerbé est affiché avec ostentation et à des endroits-charnière et dont les reportages tendaient à encourager le conflit entre les cultures.

Ses déplacements incitent $\mathrm{N}$. Jacques non seulement à repérer à l'étranger des manifestations de germanophobie mais plus encore à les provoquer et à les orchestrer. Chaque fois qu'il les signale, il les compense en rappelant son propre attachement à la culture allemande, autant par prudence qu'en raison de son prosélytisme de Luxembourgeois devenu Allemand de cœur. Par exemple, quand il recopie et traduit un article élogieux qu'il lit sur Liebknecht dans Bonnet rouge $e^{29}$, il y réplique de façon sarcastique, probablement pour se dédouaner et éloigner de lui tout soupçon, comme s'il était sur la défensive. Ou encore il fait l'éloge de "l'âme » allemande », adoptant un essentialisme possiblement hérité de Paul de Lagarde pour qui la germanité était fondée sur "l'âme" plutôt que la pureté d'une race germanique. Il emploie des sarcasmes contre toutes les confessions religieuses et peut-être l'antisémitisme de Lagarde ne lui est-il pas non plus étranger car il se moque de l'âpreté au gain qu'il prête à des commerçants juifs qu'il croise.

Sa volonté de propagande se manifeste quand il s'intéresse à l'imaginaire social des étrangers et à leurs représentations négatives de l'Allemagne. Il proclame son irritation face à l'incompréhension dont l'Allemagne ferait l'objet. Pour lui, le Kaiser serait à tort traité en responsable de la guerre. Il est aussi profondément blessé par les caricatures des cartes postales ${ }^{30}$ que l'on pourrait acheter dans tout Paris. Ce qu'il leur oppose est un relais de la propagande allemande, centrée quant à elle sur l'invincibilité des troupes, ainsi que des explications ethnographiques héritée de la psychologie des

\footnotetext{
${ }^{26}$ "Deutschland», in Gerhard HIRSCHFELD, Gerd KRUMEICH, Irina RENZ, Enzyklopädie Erster Weltkrieg, Paderborn, F. Schöningh, 2009, p. 15-30, ici p. 19.

${ }^{27}$ Cela valait également pour la presse éditée en interne pour les soldats, cf. Anne LIPP, Meinungslenkung im Krieg. Kriegserfahrungen deutscher Soldaten und ihre Deutung 19141918, Göttingen, Vandenhoeck \& Ruprecht, 2003, p. 18 et 89.

${ }_{28}$ Selon une expression qui avait été forgée en 1915 par Hermann KELLERMANN dans un ouvrage regroupant des sermons, des discours, des appels, cf. Sylvia PALETSCHEK, " Tübinger Hochschullehrer im Ersten Weltkrieg : Kriegserfahrungen an der "Heimatfront" Universität und im Feld ", in Gerhard HiRSChFELD (dir.), Kriegserfahrungen. Studien zur Sozial- und Mentalitätsgeschichte des Ersten Weltkriegs, Essen, Klartext, 1997, p. 83-106.

29 JACQUES, London und Paris, p. 174.

${ }^{30}$ Cf. Peter FisCHER, « Die propagandistische Funktion von Bildpostkarten im Ersten Weltkrieg », in Siegfried QUANDT, Der Erste Weltkrieg als Kommunikationsereignis, Gießen, Justus-Liebig-Universität, 1993, p. 63-75.
} 
peuples à la manière de Lagarde ou Langbehn, attribuant ces représentations négatives à des carences collectives: les Français seraient incapables de supporter l'idée même de la défaite, leurs " nerfs » seraient trop fragiles, et cela les conduirait au déni de la supériorité militaire allemande, déni qui proviendrait de la crise culturelle que les Français traverseraient et que N. Jacques prétendait déjà diagnostiquer en 1911. Leur dégénérescence, selon lui, reposerait moins sur des connotations biologiques que culturelles, et nulle part il ne parle de « race » française ou britannique.

Son éloge obsessionnel de la préséance culturelle allemande contamine même ses descriptions de paysages, aussi bien les vastes horizons gelés et brumeux de Podolie que les collines de Bourgogne. À preuve le paysage qu'il décrit lors de son passage à Dijon :

[Dijon] Vor der Préfecture stehen einige Dutzend Soldaten. Ein Automobil verläßt den Hof. Generale sitzen drin. Ein Soldat sagt: "Der Kommandant!" Das Automobil rast zur Stadt hinaus. Nach Westen. Die Hügel Burgunds erheben sich wellig. Die Forts sitzen unsichtbar drin, wohin das Automobil des Kommandanten rast. Die Seine hat ihre Quelle in einem der Täler dort und ihr Denkmal, und François Rude, "der große Sohn der Stadt", hat zusammen mit Noisot, "grenadier de l'île d'Elbe", auf einem dieser Hügel jenes rätselhafte Denkmal Napoleons errichtet, auf dem der Kaiser, aus langem Schlaf erwachend, sich halb im Bett hochhebt und versucht, wieder Frankreich entgegen aufzuerstehen. Aber von Dijon aus hat einmal ein germanisches Genie die französische Kunst befruchtet, der Bildhauer Klaus Sluter. Im Museum könnte man sein Grabdenkmal Philipps des Kühnen sehen, wenn es Frankreich in dieser Zeit um Kunst zu tun wäre und es seine Museen offen hielte. Dies Grabmal ist eines der erhabensten und mächtigsten Kunstwerke, die in Frankreich entstanden sind, und wurde in der Karthause von Champmol von Franzosen in der Revolution, ohne alle jene Gründe zerstört, aus denen deutsche Granaten heute gezwungen sind, die militärischen Stellungen, die das französische Heer auf Kirchtürmen einrichtet, zu beschießen. ${ }^{31}$

Le quotidien de la guerre (une des rares automobiles transporte un officier de haut rang) se conjugue ici avec la localisation géographique (la source de la Seine) et les détails historiques et artistiques. Mais N. Jacques donne un sens particulier à sa description puisque, au terme des méandres d'un paysage virtuellement reconstitué à partir du trajet imaginaire de l'automobile, elle débouche sur la justification de la guerre telle qu'elle était menée par les Allemands : les Français révolutionnaires avaient détruit leur propre patrimoine bourguignon alors que les Allemands, eux, auraient maintenant de bonnes raisons pour pilonner les clochers français, ils y seraient " obligés », autrement dit ils mèneraient une guerre défensive.

Les termes du conflit tels qu'il les pose sont d'une part le déni de la supériorité militaire allemande, supériorité qu'il entend démontrer par ses observations dont il prétend qu'elles donnent des preuves matérielles. Les termes du conflit sont d'autre part les rumeurs à propos de la cruauté et des

31 JACQUES, London und Paris, p. 206. 
exactions des soldats allemands (dont on a effectivement la trace sur les cartes postales d'époque), que N. Jacques conteste avec vigueur, accusant ceux qui les colportent d'être quant à eux incapables d'en fournir des preuves matérielles. Par ses répliques à ces agressions verbales, N. Jacques transpose chez les civils la propagande officielle des autorités gouvernementales de l'Allemagne.

Ses cibles principales sont les journalistes étrangers, si bien qu'il témoigne d'une autre guerre, celle que se livraient les médiateurs culturels des pays belligérants. Cela fait de sa part l'objet d'une réflexion synthétique, placée au début de Die Flüchtlinge. N. Jacques s'y arroge le devoir de déconstruire le discours des journalistes hostiles à l'Allemagne et de démontrer que les autres opèreraient désinformation et intoxication du public. Pour ce, il procède à un montage et met en parallèle les événements militaires et les informations données par les journaux belges, cette comparaison faisant ressortir un décalage flagrant et, selon Jacques, les mensonges de ses homologues belges. Mais le lecteur n'a alors aucune marge de manœuvre et n'est que le spectateur de cette confrontation de parole contre parole, slogan contre slogan.

$\mathrm{Si}$, à notre tour, nous sélectionnons un de ses exemples et comparons un article paru dans l'Écho de Paris du 10 décembre 1914 avec la traduction et le commentaire de N. Jacques, on voit comment il conçoit un dialogue interculturel :

[Der heftige und schmähende Artikel vom Écho de Paris, 10-12-1914] spricht mit unverkennbarer Deutlichkeit für den Geist, der Frankreich jetzt beherrscht. Er verträgt keinen weiteren Druck mehr. Er genügt sich in seiner einfältigen, begrenzten und blinden Selbstgefälligkeit. Widerstand ist für ihn schon Sieg. Und von Widerwärtigkeiten will er nichts wissen. Soviel zusammen ertragen die Nerven nicht. ${ }^{32}$

Le choix de $\mathrm{N}$. Jacques porte sur un article de propagande rédigé par Clément Garapon et intitulé «Les pessimistes ». Garapon étant le chef des services politiques de l'Écho de Paris, son point de vue était barrésien, nationaliste et militariste. Dans sa réplique, N. Jacques adopte une position tout à la fois opposée et proche, si bien que le dialogue devient impossible. Chaque propagandiste se replie sur son camp et son nationalisme, Jacques recourant aux élucubrations psychologiques du darwinisme social alors que Garapon développe des arguments politiques, visant les pacifistes, et suggère que l'ennemi véritable (et commun aux autres États) serait l'Angleterre.

Pour N. Jacques, de tels conflits verbaux font partie de son aventure. Voyager en temps de guerre, c'est une prise de risque comme il l'affectionne. Il se pare du rôle d'agent secret, signale qu'il a été sur le point d'être démasqué à plusieurs reprises et s'en amuse. La relation de son voyage en Angleterre et en France se termine comme un thriller puisque, une fois de retour chez lui et à l'abri, il lit un entrefilet du Figaro prévenant que

32 Ibid., p. 155. 
«l'Allemand Norbert Jacques » est un espion. Son triomphe est alors double : il a choisi la Suisse comme lieu de résidence, un espace neutre où il peut dire ce qu'il pense, et le Figaro lui fait le plus grand compliment qu'il puisse souhaiter en le qualifiant d'Allemand.

Enfin, ces expéditions en direction du front et la griserie de situations extrêmes étaient susceptibles de galvaniser une autre forme de sensibilité, celle qui relevait du pessimisme culturel et de la crise que les consciences avaient traversée dans la période d'avant $1914^{33}$. N. Jacques en est également un exemple significatif : la guerre lui a fourni l'occasion de prouver le bienfondé de son choix d'avoir quitté le Luxembourg en 1901 et opté pour la culture germanique.

Son évocation du conflit militaire semble annoncer Jünger, la médiation passant sans doute par Moeller van den Bruck. Son esthétisation de la violence se place encore sous le signe d'un pathos expressionniste: N. Jacques décèle dans cette "nouvelle guerre » une bestialité d'un type nouveau, la propagande belliciste des Anglais, qu'il observe en octobre 1914, serait celle d'une bête féroce : l'Angleterre qui aurait «flairé le sang ${ }^{34} »$ serait guidée par la volonté de détruire l'Allemagne, elle menacerait d'être aussi cruelle qu'elle l'aurait été pour les Boers, n'épargnant ni les femmes ni les enfants. Et la vision conclusive de son reportage sur Londres est celle d'une chaudière à vapeur, vision probablement inspirée par la salle des machines du bateau qui le ramène sur le continent, chargée des menaces inhérentes à la modernité industrielle et vision prémonitoire de la destruction du Lusitania. Une telle énergie maléfique, un tel bouillonnement démoniaque relèvent de fantasmagories dignes du lyrisme expressionniste tel qu'on le trouverait chez Walter Hasenclever. Mais, dans ce reportage, la finalité de ces allusions pathétiques n'est pas esthétique, elle reste fixée sur le «Feindbild», sur la conviction que le principal ennemi serait britannique.

La dernière description, celle du conflit militaire sur le front de l'Est (In der Schwarmlinie des österreichisch-ungarischen Bundesgenossen), est la plus aboutie sur le plan de l'écriture. Il s'agissait pour N. Jacques de traduire la violence brute qui ferait l'essence de cette guerre, sa force est perçue dans sa globalité, comme une concentration « cosmique» de «folie et de rage» :

Immer hartnäckiger knackert das trockene Gewehrfeuer. Die Geschütze brüllen bald hohl, wie kosmische verrückte Pfropfenzieher, die aus einer Weltflasche ein ganzes Gebirge herausreißen; bald schreien sie hell und grell, wie Kinder, die Angst in einem Wald überfällt. Wir fühlen, wie sich Kraft und Stahl, Feuer, Mut, Blut, Menschen und Schlamm, Raserei und kalte Wucht dort mischen, nahe bei uns, wo die Schlacht im Nebel rast. ${ }^{35}$

33 PALETSCHEK, « Tübinger Hochschullehrer », p. 168 et suiv.

34 «England hat Blut gerochen. Wehe !» (London und Paris, p. 27), écrit-il à deux reprises.

${ }^{35}$ JACQUES, Schwarmlinie, p. 44-45. 
C'est pourquoi les ennemis sont rarement approchés de façon individualisée. Les soldats morts dont il décrit le cadavre ne répondent pas à une attirance pour le morbide, les descriptions sont sobres et rappellent plus la saisissante tristesse du dormeur du val de Rimbaud qu'une complaisance malsaine pour le macabre. Le premier groupe de prisonniers russes qu'il voit nous rappelle une sculpture de Barlach :

Sie standen da unter dem trübseligen Licht, in ihren Mänteln in Regen und Schmutz, Kinder dieses Schlammes, der der Erde näher ist als der Mitteleuropäer. Kaum bewegten sie sich, große starke Männer waren es, sie erhoben sich dort in der Düsternis, wie Geister, die aus dem kotigen Boden aufgestiegen. Stumpf und schön, gewaltig gefeit vor Unglück, ein jeder selber Fatum; sie standen da mit der selbstverständlichen Teilnahmlosigkeit eines Tieres. $^{36}$

Ces prisonniers russes vus comme une masse argileuse sont décrits dans leur matérialité, statufiés, hébétés, et pourtant «beaux ». Comme si la guerre faisait revenir aux causes premières, au mythe de la genèse, de la création du genre humain, quand Yahvé modela l'homme avec la glaise du sol. Ensuite, tout au long de ce reportage en Podolie, la guerre reste présentée comme un amalgame de glaise et de mort, «Lehm und Tod». Or, comme dans le cas des prisonniers russes, cette alliance du sang et de la terre fait de la guerre un rituel de renouveau, une expression élémentaire du cycle de la vie, qui rappelle les commentaires faits par exemple par Langbehn qui décelait dans les brumes et paysages enneigés peints par le nordique Rembrandt, l'éducateur, « une mort pleine de vie».

Le sang est un autre leitmotiv qui, à première vue, s'inscrit dans la tradition d'une violence purificatrice à laquelle avaient aspiré Langbehn ou Spengler. Chez N. Jacques aussi, l'image de la purification des esprits et l'héroïsme militaire engendreraient à terme un progrès de la civilisation. Toutefois, il se limite à cette césure bénéfique que la guerre apporterait, il n'invoque en revanche pas le sang pour faire l'éloge des racines rurales ou d'une prétendue pureté de la race et il s'écarte à cet égard de l'approche mystique que l'on trouve chez Lagarde ou Langbehn. Il se borne à greffer sur ce leitmotiv sa vision de l'histoire et du progrès de la culture :

Das Friedensbündnis, das einem im Krieg zusammengeschweißten Verhältnis zwischen Völkern folgen wird, muss auf gegenseitige Waffentüchtigkeit gebaut sein, damit es gut sei. Der Krieg darf im Krieg nicht stehen bleiben, sondern muss die Blutsaat und die Bluterneuerung der Völker, die sich in ihm bewahrt haben, für eine lange Friedenszeit fruchtbar machen. ${ }^{37}$

Ces phrases qui figurent dans l'introduction de ce reportage sur le front de l'Est associent une nouvelle fois les élans esthétiques à une critique d'ordre politique que notre journaliste ne perd pas de vue ; référence est faite ici -

\footnotetext{
36 Ibid., p. 46.
}

${ }^{37}$ Ibid., p. 7. 
implicitement - à un tract que le pacifiste Umfrid avait écrit en octobre 1914 et où il était annoncé que la « vraie paix entre les peuples serait la moisson qui naîtrait de la semence sanglante du présent ${ }^{38} »$. Jacques reste journaliste, son objectif reste celui d'un nationaliste. Il justifie le bien-fondé de l'alliance avec l'Autriche- Hongrie, plaidant ainsi sans doute contre les Allemands qui émettaient des réserves à propos de cette alliance. Certes, les populations de la monarchie seraient culturellement contrastées, il est le premier à en convenir, mais les soldats de ces divers peuples slaves seraient très courageux, à commencer par les Hongrois.

Dans ce reportage, le contact entre les cultures est donc inversé par rapport aux deux précédents : ce n'est plus le conflit, mais la coexistence qui serait pittoresque, l'armée autrichienne saurait être pragmatique et utiliser les peuples, les Hongrois s'exalter de la musique des tsiganes et les Viennois imiter l'humour des juifs. Une évolution se produit chez N. Jacques : si la mentalité française lui avait paru traduire une dégénérescence, les populations slaves seraient à observer en fonction d'autres paramètres. Le partage du quotidien des soldats tirailleurs près de la ligne des tranchées se transforme au fil des pages en expérience d'autres formes de civilité, qu'il qualifie de « quasi idylliques » ${ }^{39}$, qualificatif surprenant puisqu'on est en pleine guerre mais approprié à transmettre son opinion, celle qu'un « homme nouveau » pourrait émerger.

Au demeurant, civilité obligeant, N. Jacques pondère ces positions, évite d'exacerber le goût de la violence, note sans déplaisir qu'il reste aussi des pratiques de guerre à l'ancienne, à côté de la technologie moderne. Et surtout les soirées des officiers sont agrémentées de musiques tziganes, arrosées de champagne hongrois et de Schnaps, et $\mathrm{N}$. Jacques de s'adonner à l'étrange fascination que la musique et le regard d'un tzigane exercent sur lui et sur un officier présent. Le bénéfice qu'il retire de ce séjour est son instrumentalisation de la multinationalité de l'Autriche-Hongrie dont il cautionne l'alliance avec l'Allemagne. Pour démontrer que ces soldats possèderaient le trait culturel de la virilité - attendu des combattants -, il s'intéresse à leur aspect physique et s'inscrit ainsi dans une autre des formes de la propagande durant la Première Guerre, qui a consisté à photographier les combattants, leurs silhouettes et leurs visages, et, dans la continuation de la Jugendbewegung ${ }^{40}$, à privilégier la représentation d'une jeunesse virile de manière à suggérer la convergence de qualités physiques et morales, d'endurance et d'obéissance, et de glorifier ainsi le sens du sacrifice.

\footnotetext{
38 «Ein Denkmal für die Gefallenen », cf. Karl Holl (dir.), Der deutsche Pazifismus während des Weltkrieges 1914-1918. Aus dem Nachlass Ludwig Quiddes, Boppard, Boldt, 1979, p. 50.

39 JACQUES, Schwarmlinie, p. 195.

${ }^{40}$ Cf. Jürgen ReUleKE, «Vom Kämpfer zum Krieger. Zur Visualisierung des Männerbildes im Ersten Weltkrieg », in QUANDT, Der Erste Weltkrieg als Kommunikationsereignis, p. 158175.
} 
La véritable héroïne, chez Jacques, ce n'est pas la guerre, c'est la culture germanique et celle de ses alliés. La propagande de ses feuilletons est celle d'une guerre d'opinions, dans laquelle il est lui-même un acteur engagé, qui proteste et souhaite déclencher des réactions. Elle répond aussi à son souhait personnel de mettre à mal une arrogance qu'il aurait prêtée aux Français et aux Anglais dès avant la guerre et de confirmer a posteriori son dénigrement du Luxembourg.

En transposant le conflit militaire en affrontements intellectuels, il occulte les buts de guerre. Ses écrits des années 1914-1916 ont été ceux d'un agitateur qui a cherché à attiser les conflits culturels en déconstruisant les prises de position d'autres journalistes et en apportant des rectifications chronologiques et quantitatives. Il a cherché aussi à séduire les lecteurs en variant le ton à l'intérieur de ses articles et en faisant alterner élans emphatiques et indignés, déclarations de principe, relation d'incidents ou de rencontres avec des anonymes. Capable de transmettre son goût de l'aventure et de stimuler des émotions fortes autant que de susciter l'approbation de militaires, il a prolongé le conflit sur la scène médiatique, déclenchant des répliques du Figaro et des réprimandes de politiciens luxembourgeois. $\mathrm{La}$ réception que ces écrits ont rencontrée l'a pourtant fait évoluer : ses premiers reportages récoltèrent moult éloges de la part de la presse allemande, ces articles ayant été interprétés comme actes patriotiques et les milieux militaires s'étant estimés compris et soutenus, ainsi que c'est signalé en couverture de l'édition du livre. Mais ce sont les amis pacifistes de N. Jacques qui lui adressèrent de sévères reproches, si bien qu'il préféra se réconcilier avec Schickele et publia des textes de fiction jusqu'à la fin de la guerre.

\section{La question de la paix ou les perspectives d'une autre réalité}

Les feuilletons du Berliner Tageblatt et de son supplément culturel Der Zeitgeist, quand ils abordent le sujet de la guerre, se caractérisent au contraire par leur attention à préserver les principes du droit, le sens de l'humanité et le respect d'autrui, et ce sans offenser les valeurs patriotiques, conformément à l'orientation libérale de gauche de ce quotidien et du public bourgeois et cultivé qui le lisait ${ }^{41}$. Ce journal qui avait été fondé en 1871 par Rudolf Mosse et restait conscient de son rôle dans la presse de la capitale, disposait de renseignements provenant de très bonne source qu'il plaçait à la une. Il avait des correspondants dans les grandes capitales, y compris pendant la guerre, si bien qu'il publie, dans les feuilletons, des reportages sur la Russie, la Bulgarie, la Roumanie, l'Italie. On trouve en 1916, année que nous avons regardée de plus près, beaucoup de reportages de guerre. Tous

41 KosZYK, Die Pressepolitik im Ersten Weltkrieg, p. 112-134. 
s'accompagnent, d'une façon ou d'une autre, d'une mise à distance intellectuelle et d'une rationalisation des conflits culturels.

Par exemple le 3 septembre 1916 (par conséquent en pleine offensive de la Somme), le Berliner Tageblatt publie vingt aphorismes humoristiques signés du talentueux critique littéraire Oskar Blumenthal (1852-1917), qui fut longtemps responsable de cette rubrique des feuilletons et dont le ton est en accord avec l'orientation du périodique :

Aus dem Munde einer Mutter: "es gibt keine stillen Stunden mehr - die Toten sprechen zu laut”. Der Krieg hämmert Altruisten. Oder lebt in Europa wirklich noch jemand, der sich für sich selbst interessiert?

Sans inciter à la désertion, Blumenthal refuse de cautionner un bellicisme aveugle ou un nationalisme exacerbé, quelle que soit la nation qui les pratiquerait :

Die Sparsamkeitsbewegung in England zeigt sich auch darin, dass man in den Londoner Witzblättern nur noch die billigsten Scherze findet. ${ }^{42}$

Mais une question se pose au vu de l'escapisme littéraire de certains articles : serait-il le signe que les journalistes tabouisaient la mort des soldats et la cruauté des combats ? Serait-il une forme de propagande pour protéger, rassurer la population civile ? C'est une interrogation que peut par exemple soulever une série de reportages de guerre publiés dans le Berliner Tageblatt à partir du $1^{\text {er }}$ septembre 1916 et dus à un archéologue, Wladimir Sis (18891958). Pour ce dernier, les soldats allemands qu'il a vus auraient un comportement exemplaire, donneraient du pain aux enfants affamés, ne harcèleraient pas les villageoises, bavarderaient avec les prisonniers et ne leur déroberaient pas leur nourriture. Or une telle description de la guerre a été prisée par le public berlinois, aux dires de la rédaction du journal qui se réfère au courrier de ses lecteurs. Nous pourrions conclure de cette configuration que, plus qu'une propagande, il pourrait s'agir d'une incitation à moraliser la gestion des combats.

C'est la même interprétation que nous inspire la série «Bilder aus dem Kriege » de l'Alsacien Oskar Wöhrle (1890-1946), sous titrée, comme c'est souvent le cas, par la précision que l'auteur est enrôlé ( im Feld »). Le 21 août 1916, le canonnier relate une scène idyllique vécue en Lituanie :

Ich glaubte zu träumen, als ich ins Zimmer geführt wurde. Spiegel an den Wänden, bunte Bilder, Abklatsch des Lebens, einen Tisch, ganz weiß gedeckt, blinkendes Geschirr, Polsterstühle, für einen Soldaten, der frisch aus dem Graben gekommen, Sachen, die er nur noch vom Hörensagen kannte. Die Liebenswürdigkeit der Wirte war nicht zu erschöpfen, das Gespräch floss so ruhig dahin wie das Wasser in einem sauber abgestochenem Kanalbett; das Essen war so gutmundend, dass ich keinen Augenblick daran dachte, unter fremden Menschen zu sitzen. Als die Lichter angezündet wurden, setzt (sic) sich

42 Ibid., p. 111. 
der dicke Pfarrherr an den Flügel und von einer weichen Frauenstimme getragen, schwebte das Lied durchs Zimmer.

Als ich $\mathrm{sa} ß$ beim Mütterchen, blieb die Arbeit ungetan, hatte mir mein Gärtlein mit grünem Ahorn umzäunt [...]

Einfach die Worte, einfach die Weise, Ausdruck der Seele eines Volkes, das bisher das Leben einer Pflanze geführt. ${ }^{43}$

Le fait de citer ce chant populaire lituanien, d'en vanter la simplicité, d'y déceler "l'âme d'un peuple » rappelle forcément Herder et sa collecte de chants populaires, ainsi que celle des romantiques Arnim et Brentano. Ce genre de reportage et sa publication dans le Berliner Tageblatt montrent une culture préservée en temps de guerre. La vision du peuple est certes idéalisée, aseptisée, mais elle prouve que Wöhrle préférait reproduire un « reflet de la vie » (« Abklatsch des Lebens ») que le vacarme des armes.

À la différence d'autres reportages, les journalistes du Berliner Tageblatt attirent volontiers l'attention sur les individus plus que sur le collectif. Par exemple, le 8 septembre 1916, le Berliner Tageblatt publie la relation «Der Sprüchleingraben », la vie dans les tranchées est décrite non pas avec des hommes mais avec les petites inscriptions qu'ils ont laissées. L'auteur est Wilhelm Hegeler (1870-1943), un reporter que le journal avait envoyé sur le front nord-est :

Dieser Graben an der Düna nun trug noch einen ganz besonderen Schmuck. Er war von Inschriften bedeckt wie ein mittelalterliches Haus. Er war ein rechter Sprüchleingraben. Was immer das Soldatenherz bewegt, Kampf und Sieg, Heimat und Feind, Essen und Schießen, Dienst und Freiheit, das alles war mit feinen oder derben, spaßhaften oder ernsten Worten in ein paar Reime zusammengepresst. Einer, dem die Gedanken sich vielleicht unwillkürlich zum Reim verflechten, mochte damit angefangen haben. Flugs machte ein anderer es ihm nach. Der Dritte und Vierte wollte nicht zurückstehen. So wurde ein Sport daraus. Machte sich der Telephonist ein gereimtes Hassschild über seinen Unterstand, flugs verfertigte sich auch das Maschinengewehr eine ebensolche Visitenkarte und schrieb, um allen Irrtümern vorzubeugen :

Die beste Waffe, die wir haben, ist das M. G. im Schützengraben. Sogleich meinte auch der Infanterist :

Schützen im Flankierungsgraben. Ein richtiges Visier muss jeder haben. Schießt nicht in Luft und Dreck. Es gibt dem Feinde Mut und hat keinen Zweck.

Aber mit dem Schießen ist es nicht ganz so schlimm. In ruhigeren Zeiten darf der Russe schon einmal seine Nase aus dem überdeckten Graben hervorstrecken, um Luft zu schnappen. Nur wenn er sich frech aus der Brüstung zeigt, wird sogleich gefunkt. Respekt verlangt der deutsche Soldat unbedingt vom Feind. Im übrigen aber ist die gutmütige Denkart für ihn charakteristisch, die sich in dem Vers ausprägt :

Schon den, der wehrlos sieht,

Haue den, der widersteht. Alles hat sein Spruchband gefunden, die Menschen wie die Dinge. Auf dem Kasten, in dem die Handgranaten liegen, steht : Gut ist, wenn in Kasten liegt ;

Schlecht ist, wenn in Fresse fliegt. Und vom Gasangriff heißt es : Gut ist, wenn in Lampe glieht; Schlecht ist, wenn in Nase zieht.

43 Oskar WÖHRLE, « Bilder aus dem Kriege », Berliner Tageblatt, 21 août 1916. 
[...] Alle sind sie zu Wort gekommen, der Skeptiker wie der Enthusiast, das Weltkind wie der Fromme, der Ernste und der Schalk. Dieser findet sein Kernsprüchlein im Wilhelm Busch, jener im Propheten Sirach. ${ }^{44}$

Nous observons une fois de plus que ce journaliste s'adresse à des lecteurs lettrés, capables de sourire des allusions littéraires et de priser les rimes populaires. Sa démarche est phénoménologique : son reportage illustre la variété des individus, reconstitue l'esprit, la vivacité, la créativité de chacun. Chacun aurait gardé son sens de l'humour et un art de vivre conforme au rôle que l'armée lui a affecté. La guerre des mots est transmuée en une communication ludique et spirituelle. Mais W. Hegeler ne fait pas oublier la guerre, au contraire il souligne ainsi le courage et l'endurance de ces soldats.

Cette attention à porter aux individus est problématisée dans la recension d'un recueil de lettres envoyées du front (Feldpostbriefe). En soi, une telle publication n'avait rien de spectaculaire puisque ce genre de lettres commençait à paraître en grand nombre, aussi bien dans les divers journaux que dans des livres, et que l'état-major allemand y était favorable car ces lettres devaient apporter un démenti aux rumeurs alarmistes de découragement ${ }^{45}$. Mais le présent compte rendu mérite de retenir l'attention :

\begin{abstract}
Man hat wohl hier und da unserer klassischen Geschichtsforschung den Vorwurf gemacht, dass sie die Ereignisse mehr aus der Vogelperspektive betrachte, dass sie nur die großen Richtflächen des Völkergeschehens berücksichtige, während sie an den Erscheinungen der Volksseele achtlos vorübergehe. Die Archive mit ihren aufgespeicherten Gesandtschaftsberichten, mit ihren Denkschriften und ihren Briefsammlungen von Königen, von Feldherren, von Ministern, das sind die vorzüglichsten, ja die alleinigen Quellen, aus denen die Geschichtsschreiber schöpfen, wenn sie darstellen wollen, "wie es eigentlich gewesen ist", um an ein Rankesches Wort zu erinnern. Nun tritt aber während dieses Völkerringens ohne Gleichen in der Menschheitsgeschichte ein Faktor in die Erscheinung, wie er in dieser Gestalt und in solcher Wucht auch noch nie zuvor beobachtet werden konnte. Das ganze Volk steht in Waffen und kämpft nicht nur mit einem nicht mehr zu überbietenden Heldenmut : es denkt auch über den Krieg und seine Ursachen nach und gibt seinem Denken und Fühlen in zahllosen Niederschriften unmittelbar und beredt Ausdruck. Hier sprudelt eine neuartige Quelle für den zukünftigen Geschichtsschreiber dieser ungeheuren Zeit ; hier in diesen zahllosen Briefen, in diesen Darstellungen von Einzelerlebnissen entfaltet sich die Volksseele und deren Einwirkung auf die moralische Haltung des Einzelkämpfers wird in diesen Selbstbekenntnissen kundbar. ${ }^{46}$
\end{abstract}

Cette recension livre pour ainsi dire l'arrière-plan du reportage de W. Hegeler : il s'agit d'inciter les particuliers à réfléchir à la guerre. Le commentateur souligne ici que les individualités des soldats n'ont pas été étouffées, qu'il importe de consigner leurs témoignages pour écrire une autre histoire que celle des chroniqueurs officiels, que la scientificité, jadis tant

44 Wilhelm HEGELER, « Der Sprüchleingraben », Berliner Tageblatt, 8 septembre 1916.

45 DANIEL, Augenzeugen. Kriegsberichterstattung vom 18. zum 21. Jahrhundert, p. 76-91.

${ }^{46}$ Berliner Tageblatt, 10 septembre 1916. 
souhaitée par Ranke, ne pourrait plus se concevoir sans prendre en considération le quotidien des poilus. Le journal portant ce regard critique sur les historiens issus de l'état-major (rappelons la chronique «Aus großer Zeit »), il renferme un projet politique de démocratisation et il annonce aussi la rupture épistémologique qui consistera à inclure la «petite histoire» dans la macrohistoire, comme les historiens de la Première Guerre le pratiquent de nos jours.

Mais le Berliner Tageblatt aborde aussi fréquemment et subtilement la thématique de la paix. Profitant des hésitations institutionnelles en matière de censure, des allusions sont faites aux demandes des pacifistes d'avoir l'autorisation d'aborder la question des buts de guerre. Le numéro du 21 août 1916 informe de la requête qu'avait déposée la Deutsche Friedensgesellschaft auprès de la chancellerie afin de pouvoir exposer librement son point de vue ; la réponse de la chancellerie est publiée intégralement, avec notamment sa consigne, donnée à toutes les associations et à tous les partis politiques, de s'abstenir de toute polémique, d'encourager la ténacité dans les mentalités à l'extérieur et à l'intérieur, de ne porter aucun tort à la solidarité nationale, de ne pas donner au camp ennemi l'impression que la détermination des Allemands s'affaiblirait. Cet article est suivi, toujours ce 21 août, d'un entrefilet signalant que les nationaux libéraux de Stettin ont réclamé que la censure ne s'applique qu'aux militaires, ce qui montre l'attention que ces journalistes berlinois portaient à l'aile des nationaux libéraux, certes plus proche des conservateurs mais libérale malgré tout. Le numéro du 7 septembre 1916 va jusqu'à publier le célèbre appel des pacifistes lors de leur fondation de la Zentralstelle Völkerrecht et leur exhortation à ne pas procéder à des annexions territoriales lors de la négociation de la future paix, à respecter le doit des peuples à disposer d'eux-mêmes, faute de quoi il faudrait s'attendre à de nouvelles représailles, et à mettre un terme à la course aux armements.

Cette nostalgie de la paix se manifeste souvent dans des anecdotes anodines. Par exemple un feuilleton du 10 septembre 1916 raconte un combat aérien qui a été confortablement observé depuis un jardin, par un beau soir d'été, à quelques kilomètres du front de l'Ouest : " Der Schauflieger aus Frankreich », signé de "Kurt Küchler, im Westen ». Le narrateur voit apparaitre dans le ciel un avion militaire français. Le pilote exécute toutes sortes de loopings provocateurs et virevolte gracieusement jusqu'à ce qu'une machine allemande décolle pour intimider et réprimander l'audacieux Français, lequel se retire avec agilité vers l'ouest, non sans avoir lâché quelques rafales de mitraillette. En somme les deux avions exécutent une sorte de ballet, et ce fut seulement " un jeu », « une démonstration stupide » («törichte Spielerei ») de la part de l'avion français. La perspective est donc inversée puisque ce n'est en définitive plus un reportage de guerre mais un reportage de paix en pleine guerre. Cette étrangeté, sur laquelle Kurt Küchler (1883-1925), romancier et feuilletoniste, attire l'attention, correspond au vécu d'un civil qui donne certes tort aux provocations de l'ennemi mais en 
apprécie d'autant plus le fait qu'aucun coup de feu, à l'en croire, n'aurait été tiré par l'armée allemande.

Ailleurs, une fiction intitulée "Träume eines Friedensfreundes » met en scène un pope russe qui rêve que la paix s'instaure, et ne se laisse pas décourager malgré ses déconvenues puisque ses rêves se répètent à l'infini. D'autres scènes, souvent observées en Bulgarie ou en Roumanie, rapportent comment, même dans un salon aristocratique, le ton pouvait se durcir et les règles de la civilité basculer sitôt que le sentiment national était avivé. C'est ce que rapporte, le 30 août 1916, le romancier Ferdinand Künzelmann qui avait assisté "deux ans avant la guerre ", dans un château près de Hermannstadt, à un tel incident entre des Hongrois et des Autrichiens, tous fins connaisseurs de la France et de Paris, et une Américaine, féministe enragée. Ou, pour donner un dernier exemple, signalons que, à l'occasion d'une réédition de Goethe, un article du 26 août 1916, intitulé « Goethe in Verdun », commence par le sobre constat :

Es sind dieselben Gedanken, wo auch heute wieder unsere Gedanken weilen und doch welche Unterschiede in der Stimmung zwischen Einst und Jetzt!

seule phrase qui rappelle que, lors de sa campagne en France, Goethe était passé à Verdun et avait décrit ses rencontres. Il y a donc une différence qualitative entre le passage en force de la chronique du contre-amiral Schliepe, dont nous avons vu plus haut qu'il réquisitionnait l'héritage de Faust, et la sobriété de cette allusion au Verdun de 1792, le feuilleton du Berliner Tageblatt laissant quant à lui une grande liberté d'interprétation au lecteur.

Nous avons constaté que les activités des pacifistes étaient connues aussi bien d'un nationaliste tel que N. Jacques que des journalistes du Berliner Tageblatt. Mais ce n'est que dans une revue proche de ce cercle de Romain Rolland, Die Weissen Blätter, que les feuilletons sont explicitement hostiles à la guerre. Un reportage sur «le village détruit » d'Ulrich Rauscher (18841930) renferme à la fois un plaidoyer en faveur des milieux paysans, ruinés, et la vision apocalyptique d'une nature défigurée par le déploiement de nouvelles techniques militaires :

Wer das Dorf tötet, tötet das Land. In jedem zerstörten Dorfe lockern sich die Wurzeln des Volks. Was am stärksten ist, die Bauernschaft, unterliegt wehrlos dem Krieg, weil es ihm in der blutigen Glorie seiner wahren Gestalt begegnet. Die Natur hat sich verkleinert und gezähmt, Wälder lichteten sich und Tiergeschlechter schrumpften. Nur der Mensch Vernichter ist gleich unerbittlich geblieben wie am ersten Mordtag.

Nous sommes aux antipodes des descriptions d'A. Schalek ou de N. Jacques. La dévastation de ce village de Prusse orientale devient un

\footnotetext{
47 Ulrich RAUSCHER, « Das zerstörte Dorf », Die Weissen Blätter, janvier 1915, p. 30-36, ici p. 36.
} 
absolu, propulsé hors du temps. La représentation de la guerre n'est plus liée à un seul endroit : elle est socialisante et, au-delà du social, ce sont les principes mêmes de l'existence ici-bas qui seraient ébranlés par les capacités destructrices de l'être humain.

Si l'article de Rauscher s'inspire du genre du reportage, d'autres s'inspirent de la lettre fictive. Celle d'Annette $\mathrm{Kolb}^{48}$ a la particularité d'être adressée à un mort; la guerre lui inspire de douloureux sentiments contradictoires car, si elle se sent prête à parjurer la croyance qu'elle avait jadis en la supériorité de la culture allemande, elle sait pourtant que ce serait hors de propos en un temps où la solidarité devrait avoir priorité. Le discours littéraire lui-même est d'emblée déclaré nul et non avenu puisque seul un destinataire virtuel, décédé, pourrait légitimement écouter l'aveu de la narratrice et comprendre son déchirement. Aucun mot n'est plus innocent, aucune insouciance n'est plus de mise. Ce subterfuge littéraire est au demeurant loin de saper les fondements de la communication par le biais des médias ; au contraire, il montre que c'est bien par le truchement de tels articles semi-fictifs que la diffusion de libres opinions pourrait encore être assurée.

La mobilité a permis aux reporters de guerre de repérer des contacts entre les cultures et, dans le traitement de la représentation qu'ils en donnent, nous avons constaté qu'il existait une grande variété d'un périodique à l'autre. D'un côté ce n'est guère surprenant puisque le feuilleton était un genre qui relayait les orientations de chaque rédaction et était censé répondre aux attentes des habitués du journal ; d'un autre côté ni les uns ni les autres ne pouvaient occulter les devoirs des patriotes. Mais des gradations sont à distinguer. Il y eut des journalistes et des hommes de lettres qui étaient en quête de sensations fortes et ont affiché leur excitation en s'approchant du front, ils ont mis un point d'honneur à manifester des élans nationalistes, à «modeler de la guerre une image conforme aux yeux de la hiérarchie » ${ }^{49}$; à l'instar de N. Jacques, ces reporters, quand ils étaient confrontés à l'imaginaire social des étrangers rencontrés en France et en Angleterre, ont durci la guerre des esprits et l'ont entretenue par la guerre des mots. Mais chez d'autres, l'optimisme paraît avoir été de commande et rester dans les limites d'un choix rationnel, comme dans la Neue Freie Presse. Enfin on décèle chez d'autres encore le souhait de revenir à des relations pacifiées et au dialogue des cultures, comme cela s'exprimait dans les brefs récits du Berliner Tageblatt. Ce n'était pas la curiosité qui était le mobile des reporters, mais un devoir d'écriture qui se déclinait entre solidarité avec la collectivité, comme pour A. Schalek, ou avec des individus anonymes dont les

\footnotetext{
${ }^{48}$ Outre aux travaux de Maryse STAIBER et de Maurice GodÉ, nous renvoyons à AnneMarie SAINT-GILLE, Les idées politiques d'Annette Kolb, 1870-1967: la France, l'Allemagne et l'Europe, Bern, Lang, 1993.

${ }^{49}$ Nicolas BEAUPRÉ, Écrire en guerre, écrire la guerre. France, Allemagne 1914-1920, Paris, CNRS éditions, 2006, p. 90.
} 
consciences restaient manifestement en éveil, comme pour les journalistes du Tageblatt. 